The Prolonged Exposure (PE) for Treatment of Childhood Sexual Abuse-Related PTSD:

Do We Need to Augment It?

E.A. Hembree

Pragmatic Case Studies in Psychotherapy, http://pcsp.libraries.rutgers.edu

Volume 5, Module 2, Article 4, pp. 35-44, 07-07-09 [copyright by authors]

Commentary on Individualizing Exposure Therapy For PTSD:

The Case Of Caroline

\title{
Prolonged Exposure (PE) for Treatment of Childhood Sexual Abuse-Related PTSD: Do We Need to Augment It?
}

\author{
ELIZABETH A. HEMBREE ${ }^{\mathrm{a}, \mathrm{c}}$ \& AARON P. BRINEN ${ }^{\mathrm{b}}$ \\ a Psychiatry Department, Center for the Treatment and Study of Anxiety, University of Pennsylvania \\ ${ }^{\mathrm{b}}$ Philadelphia College of Osteopathic Medicine, Philadelphia, PA \\ ${ }^{\mathrm{C}}$ Correspondence concerning this article should be addressed to Elizabeth A. Hembree, Center for the Treatment \\ and Study of Anxiety, University of Pennsylvania, 3535 Market Street, Suite 600 N, Philadelphia, PA 19104 \\ Email: hembree@mail.med.upenn.edu
}

\begin{abstract}
Kramer's thoughtful and extremely thorough case study of treating Caroline, a survivor of childhood sexual abuse (CSA), raises several important issues. A major assumption underlying Kramer's approach with Caroline's PTSD symptoms is that the Prolonged Exposure (PE) manualized treatment of Foa and her colleagues is a necessary but not sufficient method to address the clinical needs of such a client. Thus Kramer begins his treatment of this client with other clinical components, such as an assessment of Caroline's interpersonal patterns utilizing Caspar's Plan Analysis method; custom tailoring of his therapeutic relationship with Caroline; imaginative relaxation; cognitive crisis intervention; and interventions to enhance the client's social competence. He then initiates a brief course of in vivo and imaginal exposure, following the procedures in the PE manual. In this commentary, we critically evaluate this major assumption of Kramer's and review data which argue that PE can be sufficient for treating clients like Caroline. Specifically, our commentary considers the following questions: (a) What are the effects of exposure alone versus exposure combined with other treatments for PTSD? (b) Does CSA-related PTSD require additional treatment components other than PE? (c) Is PE intolerable without additional interventions? (d) Why is length of treatment important? and (e) What are some of the issues raised by the manner in which Kramer implemented the PE treatment?
\end{abstract}

Key words: childhood sexual abuse; Prolonged Exposure (PE); posttraumatic stress disorder (PTSD); Plan Analysis

Kramer's (2009) case study of treating Caroline, a survivor of childhood sexual abuse (CSA), raises several interesting questions. Can posttraumatic stress disorder (PTSD) be treated successfully with Prolonged Exposure (PE) alone, or does the addition of other treatments enhance outcome? In particular, can chronic PTSD resulting from early repetitive interpersonal trauma be successfully treated with $\mathrm{PE}$ alone, or is there something about this type of trauma that warrants additional treatment interventions? A major assumption underlying Kramer's approach with Caroline is that PE alone cannot sufficiently address the needs of such a client. He suggests 
The Prolonged Exposure (PE) for Treatment of Childhood Sexual Abuse-Related PTSD:

Do We Need to Augment It?

E.A. Hembree

Pragmatic Case Studies in Psychotherapy, http://pcsp.libraries.rutgers.edu

Volume 5, Module 2, Article 4, pp. 35-44, 07-07-09 [copyright by authors]

that the complexities of chronic, childhood abuse-related PTSD go beyond the symptoms of the disorder, and include "interpersonal difficulties" that must be addressed with additional treatment components. Kramer's assertion is that his case study illustrates the "necessity for adapting the procedure to the client's specific needs, interpersonal schema, and patterns" (p. 17), which is particularly important for clients with Caroline's trauma history, as "rape victims, in addition to their PTSD symptomatology, may suffer from interpersonal difficulties that increase their level of suffering” (p. 17).

Accordingly, Kramer utilized Plan Analysis, a method of assessing the interpersonal patterns (or goals, and the means of reaching those goals) of a client and using this information to provide "an individually custom-tailored [therapy] relationship that suits his or her most important needs and goals” (p. 4), to enhance the outcome of PE by tailoring the therapy to his client's needs (i.e., providing a "Motive-Oriented Therapy Relationship" [MOTR]). He also utilized other techniques, including imaginative relaxation, cognitive crisis intervention, and interventions to enhance the client's social competence.

We commend Dr. Kramer for his thoughtful and extremely thorough conceptualization and treatment of this client. There is no doubt that his careful attention and intervention with Caroline led to improvement in her PTSD and depression symptoms, and to the stabilization and enhancement of her social functioning. Furthermore, we congratulate Kramer for writing such a thorough case study, as this kind of article contributes to the PTSD treatment literature and offers some interesting questions to ponder. Finally, we appreciate the opportunity to address some of these questions in our commentary.

\section{EXPOSURE ALONE VERSUS EXPOSURE COMBINED WITH OTHER TREATMENTS}

First, can outcomes obtained with exposure alone be enhanced by the addition of other components? With the exception of one very recent study, reviewed below, researchers have generally failed to show that the addition of other treatment components improved outcome obtained by exposure alone. Foa, Dancu, Hembree, Jaycox, Meadows, and Street (1999) studied the addition of stress inoculation training (SIT) to PE in the treatment of 96 female assault survivors with chronic PTSD. SIT is an anxiety management program in which the client is trained in coping skills to manage their anxiety and cope with stressful situations. Foa et al. hypothesized that the combination of PE and SIT would be a more effective intervention than either component delivered separately, reasoning that the PE would yield long-term benefits by helping the client to emotionally process the trauma, while the addition of the SIT skills would lead to rapid and better management of anxiety symptoms and stressful situations. Clients were randomly allocated to one of 4 conditions: 9 sessions of treatment via PE, SIT, a combination of the two (PE-SIT), or to a waitlist (delayed treatment condition). Results showed that the addition of SIT to PE did not enhance outcome at all relative to PE alone; in fact, PE alone yielded the largest effect size (2.13) in comparison to SIT (1.27) and PE-SIT (1.5).

In a subsequent study of 171 female assault survivors, Foa, Hembree, Cahill, Rauch, Riggs, Feeny, and Yadin (2005) compared the outcome of women treated with PE alone to those 
The Prolonged Exposure (PE) for Treatment of Childhood Sexual Abuse-Related PTSD:

Do We Need to Augment It?

E.A. Hembree

Pragmatic Case Studies in Psychotherapy, http://pcsp.libraries.rutgers.edu

Volume 5, Module 2, Article 4, pp. 35-44, 07-07-09 [copyright by authors]

treated with PE combined with cognitive restructuring. The form of cognitive restructuring used in this study focused in an a priori fashion on identifying and challenging thoughts and beliefs that are commonly found in trauma survivors with PTSD. According to emotional processing theory, the theory that underlies prolonged exposure (Foa, Steketee, \& Rothbaum, 1989; Foa, Huppert, \& Cahill, 2006), beliefs such as "the world is extremely dangerous" and "I am extremely incompetent” underlie and maintain PTSD symptoms. Consistent with the 1999 outcome study, Foa et al. (2005) found that the addition of this trauma-focused cognitive restructuring (CR) to PE did not enhance treatment outcome obtained by PE alone; the effect size for PE alone was again larger (3.2) than that for PE combined with CR (2.3). Importantly, in both studies (Foa et al., 1999; Foa et al. 2005) the majority of participants had multiple traumas in their lives in both childhood and adulthood, and approximately $50 \%$ or more in each study had histories of childhood abuse.

While other studies have also failed to find that exposure combined with additional cognitive behavioral treatment components enhances outcome relative to exposure alone (Marks, Lovell, Noshirvani, Livanou, \& Thrasher, 1998; Paunovic \& Ost, 2001), a recent study by Bryant, Moulds, Guthrie, Dang, Mastrodomenico, Nixon et al. (2008) found that adding CR to imaginal and in vivo exposure improved outcomes on measures of PTSD and depression, and yielded larger effect sizes on outcome measures relative to either form of exposure alone or their combination. They randomized 118 civilian trauma survivors with PTSD to 8 sessions of treatment with either imaginal exposure alone, in vivo exposure alone, imaginal and in vivo exposure combined, and imaginal exposure, in vivo exposure, and CR combined. Participants' traumas were either non-sexual assault or motor vehicle accidents. Bryant et al. isolated the factors unique to each condition and attempted to control for any crossover of techniques. For example, in order to preserve the distinction between the exposure conditions and the exposure combined with CR condition, Bryant et al. prohibited the therapist from asking questions following the exposure-only conditions that might encourage cognitive restructuring. This study by Bryant et al. stands out in contrast to prior studies in finding that adding trauma-focused cognitive therapy enhanced outcome of exposure alone.

\section{DOES CSA-RELATED PTSD REQUIRE ADDITIONAL TREATMENT COMPONENTS?}

Kramer's notion that additional treatment is needed to augment PE in the case of CSArelated PTSD has been posed and tested by other researchers. For example, Cloitre, Koenen, Cohen, \& Han (2002) tested a two-phase treatment for 58 women who suffered PTSD from childhood abuse. The first phase consisted of skills training in affective and interpersonal regulation (STAIR), and the second phase consisted of a modified form of PE that included imaginal exposure to trauma memories but no in vivo exposure (PE). The STAIR phase focused on building therapeutic alliance and learning and practicing emotional regulation skills and interpersonal regulation skills. Cloitre and colleagues compared STAIR/PE to a minimal attention waitlist group and found that the STAIR/PE group improved in their PTSD symptoms more than the waitlist group. They found that successful completion of STAIR and good outcomes on measures of therapeutic alliance and negative mood regulation during that phase was associated with better reduction of PTSD symptoms. Cloitre and colleagues concluded that 
The Prolonged Exposure (PE) for Treatment of Childhood Sexual Abuse-Related PTSD:

Do We Need to Augment It?

E.A. Hembree

Pragmatic Case Studies in Psychotherapy, http://pcsp.libraries.rutgers.edu

Volume 5, Module 2, Article 4, pp. 35-44, 07-07-09 [copyright by authors]

the STAIR phase facilitated the exposure phase of the treatment, but the design of their study did not actually permit a good test of this hypothesis.

Cahill, Zoellner, Feeney and Riggs (2004) addressed this point in their review of research findings regarding the need for additional treatment components before implementing exposure. On the basis of their literature review, Cahill et al. concluded that no evidence existed to support the notion that exposure is intolerable for certain subgroups of trauma survivors. They also objected to Cloitre et al.'s (2002) conclusion that the STAIR phase facilitated the exposure phase of treatment because no comparison groups of “exposure only” or exposure preceding STAIR existed. Cloitre and colleagues have conducted a subsequent study with a design that does allow a test of their hypothesis, and we await publication of their results.

To date many studies have been conducted with samples that include participants with a history of multiple traumas and complex clinical presentations. Thus far, no study has demonstrated that PTSD sufferers with such history and associated clinical presentation fare worse in the standard, short-term evidenced based treatments for PTSD such as PE and cognitive processing therapy (CPT; Resick, Nishith, Weaver, Astin, \& Feuer, 2002).

\section{IS PE INTOLERABLE WITHOUT ADDITIONAL INTERVENTIONS?}

Kramer's premise also seems to imply that exposure, as a stand-alone procedure, is intolerable for PTSD sufferers, and particularly those who have experienced interpersonal violence and sexual assault and abuse in particular. If PE was intolerable, one indicator might be an increased number of dropouts from treatment. Hembree, Foa, Dorfan, Street, Kowalski, \& Tu (2003) examined the hypothesis that treatments that include exposure will be associated with a higher dropout rate than treatments that do not include exposure by analyzing data on dropout rates from 25 controlled studies that compared different cognitive behavioral treatments (CBT) for PTSD. They found that the dropout rate for exposure alone was similar to other treatments for PTSD; indeed, the results of a meta analysis of data from over 1,500 clients indicated no difference in dropout rates among exposure therapy (20.5\%), cognitive therapy and/or stress inoculation training (22.1\%), combinations of exposure and other CBT (26.9\%), and EMDR (18.9\%). These findings are consistent with previous research about the tolerability of exposure therapy.

Another sign of intolerability would be indicated by an exposure-induced exacerbation of symptoms. Foa, Zoellner, Feeny, Hembree, and Alvarez-Conrad (2002) looked at whether initiation of imaginal exposure was linked to symptom exacerbation in a group of 76 women treated for chronic assault-related PTSD. They found that a minority of clients did experience significant increases in self-reported symptoms of PTSD, depression, or anxiety following the introduction of exposure, but that such exacerbation subsided and did not affect outcome. Furthermore, individuals who experienced such exposure-related symptom exacerbation were not more likely to dropout.

While studies like these demonstrate that exposure is an effective, efficient, and safe intervention, Kramer's concerns about the use of imaginal and in vivo exposure in the treatment 
The Prolonged Exposure (PE) for Treatment of Childhood Sexual Abuse-Related PTSD:

Do We Need to Augment It?

E.A. Hembree

Pragmatic Case Studies in Psychotherapy, http://pcsp.libraries.rutgers.edu

Volume 5, Module 2, Article 4, pp. 35-44, 07-07-09 [copyright by authors]

of PTSD (and especially in the treatment assault or abuse-related PTSD) are commonly voiced. His goal of complementing or balancing-or perhaps softening-Caroline's exposure treatment with other components reflects a common concern or belief that exposure is harsh or doesn't allow for individual client needs. Feeny, Hembree, and Zoellner (2003) suggested that the gap that exists between science (illustrated by the empirical findings discussed above) and practice in the "real world" is the result of persistent clinical myths regarding exposure therapy for PTSD. They discussed the following myths:

- Myth 1. Exposure therapy is rigid and does not allow for adjusting to individual client needs;

- Myth 2. Exposure therapy alone is not enough for the complex client often seen in routine clinical practice: Additional treatment components are necessary;

- Myth 3. Existing evidence regarding the efficacy of exposure therapy does not generalize to clinical settings in the "real world"; and

- Myth 4. Exposure therapy leads to symptom worsening and high dropout rates.

Feeney et al. addressed these myths by reviewing relevant data, such as from studies like those described above, and identifying areas that require further empirical investigation. In accord with Feeny et al., we argue that exposure therapy, as implemented and empirically supported in numerous studies, is highly applicable to the treatment of a wide range of clients with PTSD by clinicians in a variety of real-world settings.

Indeed, in the current version of the PE therapist manual, Foa, Hembree, and Rothbaum (2007) stress the importance of conducting imaginal exposure in the context of a strong therapeutic alliance and with empathy, sensitivity, and ample support for the client. The client makes the decision to engage in the exposure, and we as clinicians support and applaud approaching rather than avoiding trauma memories and related reminders. When a client does struggle with avoidance, as often happens, we revisit the exposure rationale to support their choice to confront rather than avoid trauma-related memories and situations and help them recall the reasons they are choosing to do this treatment and the lives they want to reclaim.

\section{WHY IS LENGTH OF TREATMENT IMPORTANT?}

One of the striking differences between the way PE is typically implemented and the treatment course described by Kramer is the length of treatment. In research studies and in our open (non-research-study) clinic, PE is typically administered in 8 to 12 sessions. Kramer's treatment of Caroline was delivered in 40 sessions. Is this important? Does it matter whether a treatment requires 9 sessions or 40 sessions? Is it problematic if active, trauma-focused treatment is preceded by twenty preliminary or preparatory sessions devoted to alliance building and stabilization? Kramer takes that time to do good things like ensuring the safety of the client, forming a therapeutic alliance, dealing with an eating problem, and creating the Plan Analysis. Does this matter, especially given the excellent outcome achieved by Caroline? Perhaps not in 
The Prolonged Exposure (PE) for Treatment of Childhood Sexual Abuse-Related PTSD:

Do We Need to Augment It?

E.A. Hembree

Pragmatic Case Studies in Psychotherapy, http://pcsp.libraries.rutgers.edu

Volume 5, Module 2, Article 4, pp. 35-44, 07-07-09 [copyright by authors]

Caroline's case, as she was fortunate to have the time, emotional and financial resources, and motivation to remain in treatment for a year.

But in many other settings, particularly those that serve a population that is not as inclined or able to remain in longterm treatment, this treatment format causes us much more concern. In the United States, the average length of stay in treatment is about 6 sessions (e.g., Garfield, 1994; National Institute of Mental Health, 1981), and approximately 80\% of clients cease attending before their $10^{\text {th }}$ session (Pekarik, 1991). The concern this causes is that if PTSD is the most significant or worst problem a person presents, and if they are likely to only remain in treatment for less than 10 sessions, then it seems most important to implement a treatment that will efficiently and effectively reduce the target symptoms. Also, exposure treatments for PTSD have been shown to have broad effects, well beyond amelioration of PTSD symptoms (e.g., reductions is depression and anxiety and improvement in social functioning).

\section{COMMENTS ON KRAMER'S IMPLEMENTATION OF PE}

During Caroline's treatment, Kramer encountered a fairly common response from the client when she expressed ambivalence or reluctance toward engaging in imaginal exposure. After her first session of imaginal exposure to one of her memories of sexual abuse (Session 25), she was distressed and anxious about revisiting the memories again. Yet she also demonstrated an understanding of the learning opportunities and benefits that resulted from her previous in vivo exposure. In considering and responding to her ambivalence, Kramer relied on his Plan Analysis and MOTR to guide his treatment decisions and chose to terminate the imaginal exposure after only one session, because of his concern that "for me it was very important that Caroline was not being hurt at the present moment in therapy”(p.13). While we understand and appreciate Kramer's desire to respond empathically and respectfully to his client, we would not have made the same choice to support her avoidance of revisiting the trauma memories.

Imaginal exposure is used in PTSD treatment for specific reasons and yields several benefits. First, the individual with PTSD who suffers with distressing re-experiencing symptoms often confuses remembering the trauma with reliving the experience. One goal of exposure is to help the client in differentiating or distinguishing between remembering, which is safe, and the trauma itself, which was dangerous and terrible. Second, by repeatedly revisiting the traumatic memory, the client can organize the trauma memory and narrative and gain a new understanding or more realistic perspective about what happened during and after the trauma. Third, the client learns that while his anxiety may initially increase during the imaginal (or in vivo) exposure, it does not last forever. Over successive exposures, the peak of his anxiety will decrease and he will habituate to the memory. Finally, when all of these benefits accrue, the client often feels an increased sense of competence and greater mastery regarding the memory.

By understanding and effectively communicating to the client the rationale for exposure, and discussing these expected benefits, the therapist validates the client's fear or desire to avoid and yet strongly supports the client's staying with it and providing whatever rationale or help the client needs to continue with the exposure plan. It is our experience that this can be done in a way in which the client feels supported and understood, which facilitates his or her trust and 
The Prolonged Exposure (PE) for Treatment of Childhood Sexual Abuse-Related PTSD:

Do We Need to Augment It?

E.A. Hembree

Pragmatic Case Studies in Psychotherapy, http://pcsp.libraries.rutgers.edu

Volume 5, Module 2, Article 4, pp. 35-44, 07-07-09 [copyright by authors]

willingness to continue to engage in the exposure. It is important to bear in mind Foa and colleagues' (2002) finding that while a minority of clients experienced an exacerbation of symptoms at the initiation of imaginal exposure, it was not related to outcome or dropout from treatment. Not only the client, but also the therapist must understand and trust the rationale for exposure-and this can be difficult with a distressed client.

Kramer's reliance on Plan Analysis and the associated MOTR at this juncture in Caroline's treatment led him to interpret her discomfort with imaginal exposure and her anxiety about doing it again as her fear of abuse being expressed in the therapeutic relationship by cognitions such as "I feel like a 12-year-old child, helpless when alone with a man." His MOTR-guided response was to decide not to do more imaginal exposure so that "a new, positive and supportive emotional experience could be created, replacing the hitherto dysfunctional relationship patterns” (p. 17). A response guided by the emotional processing theory that underlies PE is very different.

A response that is consistent with emotional processing theory and consistent with PE would be to validate and normalize her urges to avoid by saying:

Caroline, your reaction makes a lot of sense. I understand your reluctance-no one wants to revisit painful memories that stir up bad feelings and distressing images. You've spent many years trying to avoid these memories. In the moment avoidance works very well to reduce your anxiety, but in the long run do the memories keep coming back? This tells us that the trauma is still unfinished business. So I really encourage you to hang in there and keep doing this. You have a chance now to connect with and emotionally process this experience while I am here to help you. And in doing so you will learn that this is NOW and not then-and be able to truly differentiate the abuse that you suffered during those years from this current situation that evokes memories and feelings associated with it. As you do this over and over, the discomfort and anxiety that you feel now will go down and you will feel better-and more in control, and less afraid of these memories being triggered. The imaginal exposure will help you to gain some perspective on what happened. As you gain this perspective, we expect to see the other distressing and disruptive symptoms go down as well. I know this will be unpleasant, but as we do it repeatedly it will become less difficult. I am available to support you as we go through the revisiting, during session and between sessions.

This is also a corrective emotional experience, and one that is consistent with the theoretical framework of PE therapy.

Ambivalence toward engaging in exposures is expected as a part of this treatment. Avoidance is part of PTSD, and avoidance is a major contributing factor in the long-term maintenance of PTSD symptoms after the trauma has ended. The PE therapist's job is to support the client, empathize with the distress and discomfort of confronting painful memories, and help her resolve this ambivalence and engage in exposure. It makes sense that beginning imaginal exposure would increase her anxiety and desire to avoid.

When Caroline started in vivo exposure, Kramer presented a clear and effective rationale for the intervention and Caroline was successful. It is unclear from the case description whether the rationale for imaginal exposure was delivered before starting this component. And then 
The Prolonged Exposure (PE) for Treatment of Childhood Sexual Abuse-Related PTSD:

Do We Need to Augment It?

E. A. Hembree

Pragmatic Case Studies in Psychotherapy, http://pcsp.libraries.rutgers.edu

Volume 5, Module 2, Article 4, pp. 35-44, 07-07-09 [copyright by authors]

instead of revisiting that rationale and encouraging her to continue, Kramer ended the imaginal exposure procedure in response to her predictable distress. Emotional Processing Theory (Foa \& Kozak, 1986; Foa \&Cahill, 2001) posits that avoiding memories and other trauma-related stimuli is what maintains symptoms of PTSD by preventing the incorporation of corrective information into the fear structure. Caroline missed an opportunity to learn that thoughts and memories are not dangerous; that remembering is not reliving; and that the extreme discomfort will decrease with time and repetition. Some in the trauma field suggest that survivors of CSA are fundamentally weakened as a result of early and repetitive trauma experienced during the developmental years (e.g. Cloitre et al., 2002). Kramer’s treatment of Caroline was guided by a view that something was different about the nature of her abuse. However, this is not in line with the theoretical basis of PE.

Finally, Kramer describes the treatment he provided as 40 sessions of Prolonged Exposure Therapy. However, Kramer primarily focused his treatment on ensuring safety for Caroline, stabilizing an eating disorder, and interpersonal therapy. He spent ten sessions developing the Plan Analysis. While these therapeutic activities are worthwhile, they are not PE. PE focuses on the treating the symptoms of PTSD and associated trauma-related symptoms through education, through two specific interventions, imaginal and in vivo exposure, and through a series of discussions in which the therapist helps the client to process and integrate all of the information that is emerging from her exposure experiences both in and out of the office. Kramer engaged Caroline in four sessions of in vivo exposure and one session of imaginal exposure out of 40. The current PE therapist manual (Foa, Hembree, \& Rothbaum, 2007) recommends 8-15 sessions of therapy; seven to fourteen sessions of in vivo exposure, which is mainly carried out as homework between sessions, and six to twelve sessions of imaginal exposure, conducted in the office under therapist guidance. Kramer's description of Caroline's treatment was Prolonged Exposure supplemented by Caspar’s (1995; 2007) Plan Analysis technique for individualized case formulation and treatment planning, and Caspar's MotiveOriented Therapeutic Relationship (MOTR). In our opinion, while Kramer employed exposure techniques in Caroline's treatment, a more accurate description might be Plan Analysis and MOTR supplemented by exposure interventions.

We again commend Dr. Kramer for his successful treatment of Caroline. It is clear that she benefited greatly from the therapy. Moreover, Dr. Kramer's efforts to summarize and report this interesting case are appreciated as well as the opportunity to comment on the interesting issues it raises.

\section{REFERENCES}

Bryant, R.A., Moulds, M.L., Guthrie, R.M., Dang, S.T., Mastrodomenico, J., Nixon, R.D., et al., (2008). A randomized controlled trial of exposure therapy and cognitive restructuring for posttraumatic stress disorder. Journal of Consulting and Clinical Psychology, 76(4), 695703.

Caspar, F. (1995). Plan Analysis. Towards optimizing psychotherapy. Seattle: Hogrefe.

Caspar, F. (1997). Plan Analysis. In T. D. Eells (Ed.), Handbook of psychotherapy case formulation (pp. 260-288). New York: Guilford Press. 
The Prolonged Exposure (PE) for Treatment of Childhood Sexual Abuse-Related PTSD:

Do We Need to Augment It?

E.A. Hembree

Pragmatic Case Studies in Psychotherapy, http://pcsp.libraries.rutgers.edu

Volume 5, Module 2, Article 4, pp. 35-44, 07-07-09 [copyright by authors]

Caspar, F. (2007). Plan Analysis. In T. D. Eells (Ed.), Handbook of psychotherapy case formulation ( $2^{\text {nd }}$ edition)(pp. 251-289). New York: Guilford Press.

Cloitre, M., Koenen, K. C., Cohen, L. R., \& Han, H. (2002). Skills training in affective

and interpersonal regulation followed by exposure: A phasebased treatment for PTSD related to childhood abuse. Journal of Consulting and Clinical Psychology, 70, 1067-1074.

Feeny, N.C., Hembree, E.A., \& Zoellner, L. (2003). Myths regarding exposure therapy for PTSD. Cognitive and Behavioral Practice, 10, 85-90

Foa, E. B., \& Cahill, S. P. (2001). Psychological therapies: Emotional processing. In N. J. Smelser \& P. B. Bates (Eds.), International Encyclopedia of the Social and Behavioral Sciences (pp. 12,363-12,369). Oxford: Elsvier.

Foa, E.B., Dancu, C.V., Hembree, E.A., Jaycox, L.H., Meadows, E.A., \& Street, G. (1999). The efficacy of exposure therapy, stress inoculation training, and their combination in the amelioration of PTSD for female victims of assault. Journal of Consulting and Clinical Psychology, 67, 194-200

Foa, E.B., Huppert, J.D., \& Cahill, S.P. (2005). Update on Emotional Processing Theory. In (Rothbaum, B.O., Ed). The Nature and Treatment of Pathological Anxiety. New York: Guilford Press, pp. 3-24.

Foa, E. B., \& Kozak, M. J. (1986). Emotional processing of fear: Exposure to corrective information. Psychological Bulletin, 99, 20-35.

Foa, E. B., Hembree, E. A. \& Rothbaum, B.O. (2007). Prolonged Exposure Therapy for PTSD: Emotional Processing of Traumatic Experiences. New York: Oxford University Press. (Translations in Hebrew, Spanish, and Japanese).

Foa, E.B., Steketee, G., \& Rothbaum, B.O. (1989). Behavioral/cognitive conceptualizations of post-traumatic stress disorder. Behavior Therapy, 20, 155-176.

Foa, E. B., \& Rothbaum, B. O. (1998). Treating the trauma of rape. New York: Guilford Press.

Foa, E. B., Zoellner, L. A., Feeny, N.C., Hembree, E.A., \& Alvarez-Conrad, J. (2002). Does Imaginal Exposure Exacerbate PTSD Symptoms? Journal of Consulting and Clinical Psychology 70(4), 1022-1028

Foa, E.B., Hembree, E.A., Cahill, A.M., Rauch, S.A.M., Feeny, N.C., Riggs, D.S., Yadin, E. (2005). Randomized trial of prolonged exposure for PTSD with and without cognitive restructuring: outcome at academic and community clinics, Journal of Consulting \& Clinical Psychology, 73 (5), 953-964

Garfield, S. L. (1994). Research on client variables in psychotherapy. In A. E. Bergin \& S. L. Garfield (Eds.), Handbook of psychotherapy and behavior change (4th ed., pp. 190-228). New York: Wiley.

Hembree, E.A., Foa, E.B., Dorfan, N.M., Street, G., Kowalski, J., \& Tu, X.. (2003). Do Patients Drop Out Prematurely from Exposure Therapy for PTSD? Journal of Traumatic Stress, 16(6), 555-562

Kramer, U. (2009). Individualizing exposure therapy for PTSD: The case of Caroline. Pragmatic Case Studies in Psychotherapy, Vol. 5(2), Article 1, 1-24. Available: http://hdl.rutgers.edu/1782.1/pcsp_journal.

Marks, I., Lovell, K., Noshirvani, H., Livanou, M., \& Thrasher, S. (1998). Treatment of posttraumatic stress disorder by exposure and/or cognitive restructuring. Archives of General Psychiatry, 55, 317-325. 
The Prolonged Exposure (PE) for Treatment of Childhood Sexual Abuse-Related PTSD:

Do We Need to Augment It?

E. A. Hembree

Pragmatic Case Studies in Psychotherapy, http://pcsp.libraries.rutgers.edu

Volume 5, Module 2, Article 4, pp. 35-44, 07-07-09 [copyright by authors]

National Institute of Mental Health. (1981). Provisional data on federally funded community mental health centers. Washington, DC: Government Printing Office.

Paunovic, N., \& Ost, L.G. (2001). Cognitive-behavior therapy vs exposure therapy in the treatment of PTSD in refugees. Behaviour Research and Therapy, 39, 1183-1197.

Pekarik, G. (1991). Relationship of expected and actual treatment duration for child and adult clients. Journal of Clinical and Child Psychology, 20, 121-125.

Resick, P. A., Nishith, P., Weaver, T., Astin, M.C., \& Feuer, C.A. (2002). A comparison of cognitive processing therapy, prolonged exposure, and a waiting condition for the treatment of posttraumatic stress disorder in female rape victims. Journal of Consulting and Clinical Psychology, 70, 867-879. 